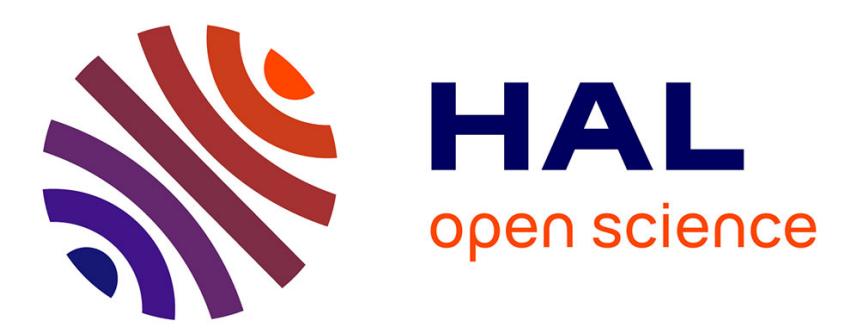

\title{
À la recherche d'un conservateur idéal : la nature versus les molécules de synthèse ? Le point de vue du chimiste Elena Giménez-Arnau
}

\section{To cite this version:}

Elena Giménez-Arnau. À la recherche d'un conservateur idéal : la nature versus les molécules de synthèse ? Le point de vue du chimiste. Revue francaise d'allergologie, 2015, 55 (3), pp.210-211. 10.1016/j.reval.2015.01.003 . hal-02119641

\section{HAL Id: hal-02119641 \\ https://hal.science/hal-02119641}

Submitted on 3 May 2019

HAL is a multi-disciplinary open access archive for the deposit and dissemination of scientific research documents, whether they are published or not. The documents may come from teaching and research institutions in France or abroad, or from public or private research centers.
L'archive ouverte pluridisciplinaire HAL, est destinée au dépôt et à la diffusion de documents scientifiques de niveau recherche, publiés ou non, émanant des établissements d'enseignement et de recherche français ou étrangers, des laboratoires publics ou privés. 
A la recherche d'un conservateur idéal : la nature versus les molécules de synthèse ? - Le point de vue du chimiste

The quest for the ideal biocide : nature versus synthetic molecules ? - The chemist's point of view Elena Giménez-Arnau

Laboratoire de Dermatochimie, Université de Strasbourg-CNRS UMR7177, Institut le Bel, 4 Rue Blaise Pascal, 67081 Strasbourg, Tel : 0368851525, E-mail : egimenez@unistra.fr

Mots clés : conservateur, allergie cutanée, origine naturelle, molécules de synthèse

Key words : biocides, skin allergy, natural origin, synthetic molecules

Les conservateurs (ou biocides) sont des substances chimiques, d'origine naturelle ou synthétique, qui sont ajoutées aux produits finis afin de les mettre à l'abri de proliférations microbiennes ou de modifications chimiques indésirables, assurant ainsi la sécurité du produit et, en conséquence, celle du consommateur. Dans les produits cosmétiques et pour les soins de la peau, les conservateurs utilisés sont fréquemment décrits comme étant la deuxième source principale d'allergies de contact après les fragrances. Aujourd'hui, 55 conservateurs sont autorisés dans les produits cosmétiques et répertoriés dans la première partie de l'annexe VI de la Directive Européenne Cosmétiques (76/768/EEC), pièce de la législation européenne qui régule leur fabrication et mise sur le marché. Ces conservateurs sont tous sujets à des restrictions en termes de concentration, dépendant du domaine d'utilisation du produit cosmétique concerné.

Pour qu'un conservateur soit « idéal » il doit répondre à une série de critères tels que : avoir un spectre large d'activité, être efficace durant toute la durée de vie du produit, être de préférence liquide et soluble dans l'eau, être efficace dans une gamme large de $\mathrm{pH}$, ne pas être désactivé par d'autres ingrédients, être sans odeur et sans couleur et, bien évidemment, être sûr.

Il existe un certain nombre, assez restreint, de conservateurs autorisés par la législation européenne d'origine naturelle. Le chlorure d'argent (20\%), par exemple, déposé sur un substrat de dioxyde de titane, est interdit dans les produits pour enfants sous l'âge de trois ans, dans les produits d'hygiène oral et dans ceux appliqués autour des yeux et des lèvres, mais est utilisé dans les protecteurs solaires et dans certains produits de maquillage. L'acide benzoïque et l'alcool benzylique sont permis à une concentration de $0.5 \%$ et de $1 \%$ respectivement et peuvent être extraits à partir de résines balsamiques. L'acide sorbique, permis à $0.6 \%$, peut être aussi rencontré dans la nature. On l'a d'abord isolé des baies immatures du sorbier (Sorbus Aucuparia), mais aujourd'hui il est essentiellement fourni par voie de synthèse.

Mis à part ces quelques composés autorisés par la législation, un cas qui mérite particulière attention est celui des huiles essentielles. Beaucoup de gens pensent que tout ce qui est naturel est bon. Or, malgré que nombreuses huiles essentielles aient des propriétés bactéricides, elles peuvent induire une sensibilisation chez les consommateurs avec le développement, en conséquence, d'une allergie cutanée (eczéma allergique de contact). II s'agit d'extraits végétaux, obtenus par extraction mécanique, 
entraînement à la vapeur d'eau ou distillation à sec. Ce sont en réalité des vrais mélanges de molécules variées, comprenant en particulier des terpènes (hydrocarbures non aromatiques), et des composés oxygénés (alcools, aldéhydes, cétones, esters). Nombreuses de ces molécules sont des sensibilisants connus comme l'aldéhyde cinnamique et l'eugénol, ainsi que les produits d'oxydation de terpènes odorants tels que le pinène, le limonène et le linalol.

Il est, en conséquence, important de bien réfléchir aux mentions indiquées sur les produits telles que "sans conservateur » versus « conservateur naturel ». Ce n'est pas parce qu'un produit est $100 \%$ naturel, qu'il ne peut pas être source de sensibilisation et de dermatite allergique de contact.

La majorité de conservateurs listés dans l'annexe VI de la Directive Européenne Cosmétiques (76/768/EEC) sont, cependant, des composés ou molécules obtenues par voie de synthèse. En effet, les produits d'origine naturelle sont souvent d'extraction difficile et couteuse. Nous pouvons citer par exemple le formaldéhyde et les libérateurs de formaldéhyde (bronopol, méthénamine, imidazolidinyl urée, diazolidinyl urée, DMDM hydantoïne, Quaternium 15), le mélange d'isothiazolinones méthylchloroisothiazolinone et méthylisothiazolinone, le phénoxyéthanol, le benzoate de sodium, ou le triclosan. Nombreux de ces produits de synthèse peuvent, cependant, induire également des sensibilisations et des allergies cutanées. La polémique engendrée par l'utilisation des parabènes (méthyl-, éthyl-, propyl-) a eu comme conséquence principale le problème de savoir par quoi les remplacer. Les alternatives font parfois "pire que mieux». Dans les années 1980 le mélange méthylchloroisothiazolinone ( $\mathrm{MCl}$ )/méthylisothiazolinone (MI) (Kathon CG, $\mathrm{MCl} / \mathrm{MI}$ 3:1) était très utilisé. Suite aux nombreux cas décrits de sensibilisation cutanée, la réglementation européenne en avait limité l'usage et les concentrations autorisées à $15 \mathrm{ppm}$. Suite à cette mesure, le nombre de cas d'allergie s'était stabilisé autour de $2 \%$ après les années 1990. Pendant ces années, le conservateur Euxyl K400, mélange de méthyldibromoglutaronitrile et de phénoxyéthanol, pris la place de $\mathrm{MCl} / \mathrm{MI}$. II se releva aussi sensibilisant principalement à cause du méthyldibromoglutaronitrile, interdit dans les produits cosmétiques en Europe depuis 2007. Les parabènes furent alors très employés mais des doutes sur leur innocuité et toxicité potentielle les ont fait disparaître. Au début des années 2000, afin de pouvoir remplacer les parabènes, la $\mathrm{MI}$, qui était considérée comme moins sensibilisante que la $\mathrm{MCl}$ dans le mélange Kathon CG, a commencé alors à être utilisée dans des produits cosmétiques et industriels (colles, peintures, encres) mais à des concentrations plus fortes que dans le mélange $\mathrm{MCl} / \mathrm{Ml}$ à cause de son pouvoir biocide plus faible. Malheureusement, à plus fortes concentrations la Ml s'est avérée être un très fort sensibilisant, produisant une explosion des cas de sensibilisation de contact en Europe qui a été qualifiée d'épidémie. La MI est aujourd'hui autorisée à 100 ppm dans les produits cosmétiques dits rincés et nonrincés, mais aucune législation n'existe pour les produits industriels. Le Scientific Committee on Consumer Safety (SCCS) de la Commission Européenne a statué dans son opinion de Décembre 2013 que seulement une concentration de $15 \mathrm{ppm}$ de $\mathrm{Ml}$ pouvait être sûre pour les consommateurs, et ceci seulement dans les produits rincés. 
A la vue de tous ces éléments, que ce soit dans le cas des conservateurs d'origine naturelle, ou dans celui des conservateurs d'origine synthétique, trouver un conservateur « idéal », avec risque zéro pour le consommateur, est une tâche extrêmement difficile et reste sans aucun doute un question prioritaire pour l'industrie.

Cosmetic Directive 2005/42/EC. Off J Eur Union 2005: L158: 17-19.

Schnuch A, Lessmann H, Geier J, Uter W. Contact allergy to preservatives. Analysis of IVDK data 19962009. Br J Dermatol 2011: 164: 1316-1325.

Svedman C, Andersen K E, Brandão F M, Bruynzeel D P, Diepgen T L, Frosch P J, Rustemeyer T, Giménez Arnau A, Gonçalo M, Goossens A, Johansen J D, Lahti A, Menné T, Seidenari S, Tosti A, Wahlberg J E, White I R, Wilkinson J D, Mowitz M, Bruze M. Follow-up of the monitored levels of preservatives sensitivity in Europe. Overview of the years 2001-2008. Contact Dermatitis 2012: 67: 312314.

Gonçalo M, Goossens A. Whilst Rome burns: the epidemic of contact allergy to methylisothiazolinone. Contact Dermatitis 2013: 68: 257-258. 